Math. Ann. 309, 483-490 (1997)

Mathematische

Annalen

(c) Springer-Verlag 1997

\title{
An inflectionary tangent to the Kummer variety and the Jacobian condition
}

\author{
Giambattista Marini \\ Dipartimento di Matematica, Università di Roma "Tor Vergata", Via della Ricerca Scientifica, \\ I-00133 Roma, Italy (e-mail: marini@axp.mat.utovrm.it)
}

Received: 5 August 1996

Mathematics Subject Classification (1991): 14H40

\section{Introduction}

In this paper we deal with a degenerate version of the trisecant conjecture (see Welters [W1]). Let $(X,[\Theta])$ be an indecomposable principally polarized abelian variety and let $\Theta$ be a symmetric representative of the polarization. We shall denote by $\theta$ a non-zero section of the sheaf $\mathscr{C}_{X}(\Theta)$. The linear system $|2 \Theta|$ is base-point-free and it is independent of the choice of $\Theta$. The image of the morphism $K: X \longrightarrow|2 \Theta|^{*}$ associated with the base-point-free linear system $|2 \Theta|$ is a projective variety which is called the Kummer variety of $(X,[\Theta])$. Welters conjectured in [W1] that the existence of one trisecant line to the Kummer variety characterizes the Jacobians (it is well known that the Kummer variety of a Jacobian has a rich geometry in terms of trisecants and flexes).

We prove that if there exists an inflectionary tangent $l$ to the Kummer variety associated with $(X,[\Theta])$, then $(X,[\Theta])$ is a Jacobian provided that there are no set-theoretical $D$-invariant components of the scheme $D \Theta:=\Theta \cap\{D \theta=0\}$, where $D$ is an invariant vector field on $X$ associated to $l$.

Observe that Welters' conjecture divides naturally into the three possible cases which correspond to the three hypotheses: $i$ ) the Kummer variety $K(X)$ has an honest trisecant, i.e. there exists a line $l$ in $|2 \Theta|^{*}$ meeting $K(X)$ at three distinct points $K(a), K(b), K(c)$; ii) there exists a line $l$ in $|2 \Theta|^{*}$ which is tangent to $K(X)$ at a smooth point $u$, and which meets $K(X)$ at some other point; iii) $K(X)$ has an inflectionary tangent at a smooth point. The cases $i$ ) and $i i)$ were considered by Debarre, see [D1] and [D2] where he gives an affermative answer to the problem, provided that an extra hypothesis holds. Namely, in case $i$ ) the extra hypothesis is that $\Theta$ cannot contain invariant divisors under the translations $t_{a-b}, t_{b-c}$, and in case $\left.i i\right)$ the extra hypothesis is that $\Theta$ 
cannot contain invariant divisors under the translation $t_{2 u}$. Here we deal with the third case. Our method consists in proving that the hypothesis iii) implies the hypothesis of Shiota's theorem on Novikov's conjecture, i.e. that the K.P. equation holds, thereby proving that $(X,[\Theta])$ is a Jacobian. It is worth noticing that the K.P. hypothesis takes naturally the fourth place in the list above, indeed it turns out to be equivalent to the hypothesis that there exixts a length 4 germ of a one-parameter family of inflecionary tangents at $K(0)$ (which is a singular point of the Kummer variety), see [AD1], or [W1] p. 499, or [W2] fact 2.18.

It deserves to be pointed out that the technique used here, for some aspects, goes back to Arbarello and De Concini and their attempt to give an algebraic solution to the Novikov's conjecture (see [A], [AD1]).

We make use of Ein-Lazarsfeld's theorem on Arbarello-De Concini's conjecture (see Corollary 2 in [EL]), which states that the singular locus of the theta divisor of an indecomposable principally polarized abelian variety has at least codimension 2.

\section{An inflectionary tangent to the Kummer variety}

We start with a proposition translating the hypothesis that the Kummer variety has an inflectionary tangent into an equation satiefied by the theta function.

Proposition 1. Let $(X,[\Theta])$ be an indecomposable principally polarized abelian variety of dimension $>1$. The following conditions are equivalent

i) The Kummer variety $K(X)$ of $(X,[\Theta])$ has an inflectionary trisecant, i.e. there exists a smooth point $K(u)$ in $K(X)$, where $K: X \rightarrow|2 \Theta|^{\star}$ is the Kummer morphism, and a line $l$ in the projective space $|2 \Theta|^{\star}$ which meets $K(X)$ at $K(u)$ with at least multiplicity 3;

ii) There exixt invariant vector field $D_{1} \neq 0, D_{2}$, a complex number $c$ and a point $u$ in $X$ such that

$$
\begin{aligned}
P \theta:= & D_{1}^{2} \theta \cdot \theta_{2 u}-2 D_{1} \theta \cdot D_{1} \theta_{2 u}+\theta \cdot D_{1}^{2} \theta_{2 u} \\
& +D_{2} \theta \cdot \theta_{2 u}-\theta \cdot D_{2} \theta_{2 u}+c \cdot \theta \cdot \theta_{2 u}=0,
\end{aligned}
$$

where $\theta_{2 u}(z):=\theta(z-2 u)$;

iii) There exixt an invariant vector field $D_{1} \neq 0$ and a point $u$ in $X$ such that the following inclusion holds

$$
\Theta \cap \Theta_{2 u} \quad \subset \quad D_{1} \Theta \cup D_{1} \Theta_{2 u},
$$

where $D_{1} \Theta$ is the scheme of zeroes of the section $D_{1} \theta \in H^{0}\left(\Theta, \mathscr{C}_{\Theta}(\Theta)\right)$.

We recall that $K(u)$ is a smooth point of $K(X)$ if and only if $2 u \neq 0$. In particular in $i$ ) it is assumed $2 u \neq 0$. On the other hand, equation 1.1 implies $2 u \neq 0$ (for otherwise $D_{1} \theta$ would vanish on $\Theta$ ), and inclusion 1.2 implies $2 u \neq 0$ for dimensional reasons. 
Proof. It is convenient to choose a basis $\left\{\vartheta_{\nu}\right\}$ of $H^{0}(X, \mathscr{O}(2 \Theta))$ having the property that Riemann's quadratic identity $\theta(z+\zeta) \cdot \theta(z-\zeta)=\sum_{\nu} \vartheta_{\nu}(z) \cdot \vartheta_{\nu}(\zeta)$ holds, and to denote by $\vec{\vartheta}$ the vector $\left(\ldots, \vartheta_{\nu}, \ldots\right)$. Property $\left.i\right)$ is equivalent to the existence of a germ of curve $c(\epsilon)=u+\epsilon \cdot D_{1}+\epsilon^{2} \cdot D_{2}$ such that the line $l(t)=K(u)+t \cdot K_{\star}\left(D_{1}\right)$ is an inflectionary tangent to $K(c(\epsilon))$ at $K(u)$ (here $\epsilon$ and $t$ are complex parameters). It follows that it is equivalent to the property that the vectors $\vec{\vartheta}(u), \vec{\vartheta}\left(u+\epsilon \cdot D_{1}\right)$ and $\vec{\vartheta}\left(u+\epsilon \cdot D_{1}+\epsilon^{2} \cdot D_{2}\right)$ are dependent modulo $\epsilon^{3}$. Thus, taking series expansion with respect to $\epsilon$ we infer that $i$ ) holds if and only if there exist non-simultanously-zero contants $c, b, a$ such that $c \vec{\vartheta}(u)+b D_{1} \vec{\vartheta}(u)+a\left[{ }_{2}^{1} D_{1}^{2}+D_{2}\right] \vec{\vartheta}(u)=0$, or rather that $\left.\left[c \vartheta_{\nu}(\zeta)+b D_{1} \vartheta_{\nu}(\zeta)+a\left[{ }_{2}^{1} D_{1}^{2}+D_{2}\right] \vartheta_{\nu}(\zeta)\right]\right|_{\zeta=u}=0$, for all $\nu$. Multiplying by $\vartheta_{\nu}(z)$, taking the sum over all subscripts $\nu$ and using Riemann's relation the equalities above turn out to be equivalent to the property that the section

$$
\begin{aligned}
& {\left[c \theta(z+\zeta) \cdot \theta(z-\zeta)+b D_{1}[\theta(z+\zeta) \cdot \theta(z-\zeta)]\right.} \\
& \left.\quad+a\left[\frac{1}{2} D_{1}^{2}+D_{2}\right][\theta(z+\zeta) \cdot \theta(z-\zeta)]\right]\left.\right|_{\zeta=u}
\end{aligned}
$$

is zero. Let us now assume that this is the case. The sections $\theta(z+u) \cdot \theta(z-u)$, $\left.D_{1}[\theta(z+\zeta) \cdot \theta(z-\zeta)]\right|_{\zeta=u}=D_{1} \theta_{-u} \cdot \theta_{u}-\theta_{-u} \cdot D_{1} \theta_{u}$ are independent because $2 u \neq 0$ (i.e. $u \neq-u$ ). It follows that $a \neq 0$, so that we can assume $a=2$. We can assume $b=0$ up to adding a multiple of $D_{1}$ to $D_{2}$ (namely ${ }_{a}^{b} D_{1}$ ). It follows that property $i$ ) is equivalent to the existence of invariant vector fields $D_{1} \neq 0, D_{2}$, a constant $c$, and a point $u$ in $X$ such that

$$
\left[c \cdot \theta(z+\zeta) \cdot \theta(z-\zeta)+\left[D_{1}^{2}+2 D_{2}\right][\theta(z+\zeta) \cdot \theta(z-\zeta)]\right]_{\zeta=u}=0
$$

which is property ii) (modulo rescaling $D_{2}$, and translating by $u$ ).

From the equation 1.1 we obtain that $D_{1} \theta \cdot D_{1} \theta_{2 u}$ vanishes on $\Theta \cap \Theta_{2 u}$, it follows that ii) implies iii). On the other hand, if the inclusion 1.2 holds, the section $\sigma:=D_{1}^{2} \theta \cdot \theta_{2 u}-2 D_{1} \theta \cdot D_{1} \theta_{2 u}+\theta \cdot D_{1}^{2} \theta_{2 u}$ vanishes on the scheme $\Theta \cap \Theta_{2 u}$. Looking at the exact sequence

$$
0 \longrightarrow \mathscr{C}_{\Theta}(\Theta) \stackrel{\theta_{2 u}}{\longrightarrow} \mathscr{C}_{\Theta}\left(\Theta+\Theta_{2 u}\right) \longrightarrow \mathscr{C}_{\Theta \cap \Theta_{2 u}}\left(\Theta+\Theta_{2 u}\right) \longrightarrow 0
$$

it follows that there exists an invariant vector field $D_{2}$ such that $\left.\sigma\right|_{\Theta}=-D_{2} \theta$. $\left.\theta_{2 u}\right|_{\Theta}$. Therefore $\sigma^{\prime}:=\sigma+D_{2} \theta \cdot \theta_{2 u}-\theta \cdot D_{2} \theta_{2 u}$, which is a section of $Q_{X}\left(\Theta+\Theta_{2 u}\right)$, vanishes on $\Theta$. Looking at the exact sequence

$$
0 \longrightarrow \mathscr{Q}_{X}\left(\Theta_{2 u}\right) \stackrel{\theta}{\longrightarrow} \mathscr{Q}_{X}\left(\Theta+\Theta_{2 u}\right) \longrightarrow \mathscr{C}_{\Theta}\left(\Theta+\Theta_{2 u}\right) \longrightarrow 0
$$

it follows that there exists a section $\eta=-c \cdot \theta_{2 u}$ of the line bundle $\mathscr{Q}_{X}\left(\Theta_{2 u}\right)$ such that $\sigma^{\prime}=\theta \cdot \eta$. This gives 1.1 .

q.e.d. 
Theorem 2. Let $(X,[\Theta])$ be an indecomposable principally polarized abelian variety. Assume that the Kummer variety $K(X)$ of $(X,[\Theta])$ has an inflectionary trisecant, i.e. that the condition ii) of proposition 1 holds, and assume that the scheme $D_{1} \Theta$ does not contain set-theoretical $D_{1}$-invariant components. Then, $(X,[\Theta])$ is the Jacobian of a smooth curve $\mathscr{C}$.

Proof. We want to prove that $\left(D_{1}^{2}-D_{2}\right) \theta \cdot\left(D_{1}^{2}+D_{2}\right) \theta$ vanishes on the scheme $\Theta \cap$ $\left\{D_{1} \theta=0\right\}$, which we shall denote by $D_{1} \Theta$. The above property characterizes Jacobians by Shiota's Theorem (see $[S]$ ). In fact, the restriction of the K.P. equation to $D_{1} \Theta$ is $\left(D_{1}^{2}-D_{2}\right) \theta \cdot\left(D_{1}^{2}+D_{2}\right) \theta$, and the K.P. equation is equivalent to its vanishing on $D_{1} \Theta$ (this is a particular case of a standard fact proved in [AD1], p. 118).

Let $W$ be a component of the scheme $D_{1} \Theta$, and let $p$ be a generic point of the underlying reduced scheme $W_{\text {red }}$. By Ein-Lazarsfeld's theorem, $\Theta$ is smooth at $p$. Hence there exist an irriducible element $h$, invertible elements $\beta, \gamma, \delta$, and elements $\tilde{\alpha}, \tilde{\beta}, \tilde{\gamma}, \tilde{\delta}$, all of them in the local ring $\mathscr{Q}_{X, p}$, and integers $m \geq 1, s, r, t$, such that the ideal of $W_{\text {red }}$ at $p$ is $(h, \theta)$, and such that

$$
\begin{array}{ll}
D_{1} \theta & =h^{m}+\tilde{\alpha} \cdot \theta, \\
\theta_{2 u} & =\beta \cdot h^{s}+\tilde{\beta} \cdot \theta, \\
\left(D_{1}^{2}+D_{2}\right) \theta & =\gamma \cdot h^{r}+\tilde{\gamma} \cdot \theta, \\
\left(D_{1}^{2}-D_{2}\right) \theta & =\delta \cdot h^{t}+\tilde{\delta} \cdot \theta .
\end{array}
$$

We can now translate the result that we need to prove in terms of the setting above. The ideal of $W$ at the point $p$ is $\left(h^{m}, \theta\right)$. On the other hand,

$$
\left(D_{1}^{2}-D_{2}\right) \theta \cdot\left(D_{1}^{2}+D_{2}\right) \theta=\gamma \delta \cdot h^{r+t} \bmod (\theta) .
$$

Thus to conclude our proof it suffices to show that

$$
r+t \geq m \text {. }
$$

Since the restriction of $P \theta$ to $W$ equals $\left(D_{1}^{2}+D_{2}\right) \theta \cdot \theta_{2 u}$, in case $\theta_{2 u}$ is invertible at $p$ we are done. Thus we can assume that $\theta_{2 u}$ vanishes on $W_{\text {red }}$. This gives $s \geq 1$. By substitution of the equalities in 2.1 into the expressions of $P \theta$ and $D_{1} P \theta$ we obtain the equations 2.2 and 2.3 below

$$
\begin{aligned}
& 0=P \theta=\left(D_{1}^{2}+D_{2}\right) \theta \cdot \theta_{2 u}-2 D_{1} \theta \cdot D_{1} \theta_{2 u}= \\
& \beta \gamma \cdot h^{r+s}-2 h^{m} \cdot \omega \bmod (\theta),
\end{aligned}
$$

where $\omega:=D_{1} \theta_{2 u}=\left[s \beta \cdot D_{1} h \cdot h^{s-1}+D_{1} \beta \cdot h^{s}+\tilde{\beta} \cdot h^{m}\right] \bmod (\theta)$;

$$
0=D_{1} P \theta=
$$

$$
\begin{aligned}
& -\left(D_{1}^{2}-D_{2}\right) \theta \cdot D_{1} \theta_{2 u}+D_{1}\left(D_{1}^{2}+D_{2}\right) \theta \cdot \theta_{2 u}+ \\
& D_{1} \theta \cdot\left[c \cdot \theta_{2 u}-\left(D_{1}^{2}+D_{2}\right) \theta_{2 u}\right]= \\
& -\delta h^{t} \cdot \omega+\left(D_{1}\left(\gamma \cdot h^{r}\right)+\tilde{\gamma} h^{m}\right) \cdot \beta h^{s}+c \cdot h^{m+s} \\
& \quad-h^{m} \cdot\left[\left(D_{1}^{2}+D_{2}\right)\left(\beta h^{s}\right)+\tilde{\beta} \gamma h^{r}+2\left(D_{1} \tilde{\beta}\right) \cdot h^{m}\right] \bmod (\theta) .
\end{aligned}
$$


Let us look at the equation 2.2. Since $\beta \cdot \gamma$ is invertible, $r+s \geq m+s$ if $\omega \in\left(h^{s}, \theta\right)$. So that, if this is the case, $r \geq m$ and we are done. We can therefore assume that

$$
\omega=D_{1} \theta_{2 u} \notin\left(h^{s}, \theta\right) .
$$

We now come to our first reduction: roughly speaking, we shall reduce our problem to the case where the order of the contact at $p$ between the hypersurface $\left\{D_{1} \theta=0\right\}$ and $\Theta$ equals the one between the hypersurface $\left\{D_{1} \theta_{2 u}=0\right\}$ and $\Theta$. More formally, our reduction shall lead us to 2.5 below. For this reduction we distinguish the two cases $m \geq s$, and $s>m$. First, assume $m \geq s$. Computing 2.3 modulo $\left(h^{s}, \theta\right)$ we obtain $-\delta \cdot h^{t} \cdot \omega=0 \bmod \left(h^{s}, \theta\right)$. This implies $t \geq 1$ because $\delta$ is invertible and $\omega \notin\left(h^{s}, \theta\right)$ (by 2.4). Looking at the definition of $\omega$, or rather looking at its expression modulo $(\theta)$, we see that our assumption $m \geq s$ implies that $\omega$ is in $\left(h^{s-1}, \theta\right)$. Then, going back to 2.2 we get $r+s \geq m+s-1$. This gives $r \geq m-1$. It follows that $r+t \geq m$ and we are done. Let us now turn to the case $s>m$, that is, since $m \geq 1$,

$$
s \geq m+1 \geq 2 .
$$

First, we observe that $\tilde{\beta}$ must be invertible. In fact, if $E$ is an invariant vector field on $X$, then $E \theta_{2 u}=E \beta \cdot h^{s}+s E h \cdot h^{s-1}+E \tilde{\beta} \cdot \theta+\tilde{\beta} \cdot E \theta$. So that, as $s \geq 2$, if $\tilde{\beta}$ would not be invertible then $E \theta_{2 u}$ would vanish on $W_{\text {red }}$ for all invariant vector fields $E$, i.e. $\theta_{2 u}$ would be singular along $W_{\text {red }}$ and Ein-Lazarsfeld's Theorem would be contradicted. Since $s>m$, the element $\omega$ is in $\left(h^{m}, \theta\right)$ by its very definition. If $s=m+1$, then $\omega \notin\left(h^{m+1}, \theta\right)$ by 2.4. If $s>m+1$, then $\omega=\tilde{\beta} \cdot h^{m} \bmod \left(h^{m+1}, \theta\right)$, so that $\omega$ is not in $\left(h^{m+1}, \theta\right)$ because $\tilde{\beta}$ is invertible. It follows that, in any case, we are allowed to write

$$
\omega=\rho \cdot h^{m}+\tilde{\rho} \cdot \theta
$$

where $\rho$ is invertible. As for the next reduction, we shall prove that (unless to conclude) the restrictions $\left.\left(D_{1}^{2}+D_{2}\right) \theta\right|_{\Theta},\left.\left(D_{1}^{2}+D_{2}\right) \theta\right|_{\Theta}$ vanish at $p$ with the same multiplicity (namely, we shall prove that we can assume 2.6 below). Substituting 2.5 into 2.2 we obtain $\beta \gamma h^{r+s}-2 \rho h^{2 m}=0 \bmod (\theta)$, it follows that $r+s=2 m$. In view of this equality, writing 2.3 modulo $\left(h^{2 m-1}, \theta\right)$ we obtain

$$
-\delta \cdot \rho \cdot h^{t+m}-\tilde{\beta} \cdot \gamma \cdot h^{m+r}=0 \quad \bmod \left(h^{2 m-1}, \theta\right) .
$$

Then, since both $\delta \rho$ and $\tilde{\beta} \gamma$ are invertible, either $r=t \leq m-2$, or $r, t \geq$ $m-1$. In the latter case, either $r=t=m-1$, or $r+t \geq m$ which is $(\star)$. It follows that, in any event, we can assume that

$$
r=t \leq m-1
$$

(for otherwise we would be done). We are now going to conclude our proof (it is worth noticing that we use here our extra hypothesis that $W_{\text {red }}$ is not 
$D_{1}$-invariant). First we prove an equality (2.7 below) which turns out to take care of the case where $\left(D_{1}^{2}-D_{2}\right) \theta$ vanishes on $W_{\text {red }}$ (this is the case where $t=0$ ); then, we give a formal argument (not using the property $P \theta=0$ ) which turns out to take care of the case where $r$, hence $t$, is up to $m-2$. Writing down $\left(D_{1}^{2}+D_{2}\right) P \theta$ and omitting the terms in $\left(h^{m}, \theta\right)$ such as $\theta, D_{1} \theta, \theta_{2 u}, D_{1} \theta_{2 u}$ we obtain $\left(D_{1}^{2}+D_{2}\right) P \theta=-2\left(D_{1}^{2}-D_{2}\right) \theta \cdot D_{1}^{2} \theta_{2 u} \bmod \left(h^{m}, \theta\right)$. Therefore, since $P \theta$ vanishes by hypothesis, substituting 2.1 and 2.5 into the equality above we obtain

$$
0=\left(D_{1}^{2}+D_{2}\right) P \theta=-2 \delta h^{t} \cdot m \rho D_{1} h \cdot h^{m-1} \bmod \left(h^{m}, \theta\right) .
$$

Now plug 2.1 into the equation $D_{1}\left(D_{1}^{2}+D_{2}\right) \theta=\left(D_{1}^{2}+D_{2}\right) D_{1} \theta$. We get

$$
D_{1}\left(\gamma \cdot h^{r}+\tilde{\gamma} \cdot \theta\right)=\left(D_{1}^{2}+D_{2}\right)\left(h^{m}+\tilde{\alpha} \cdot \theta\right) .
$$

We are now going to prove that

$$
\text { either } \quad r=0, \quad \text { or } \quad r=m-1, \quad \text { or } \quad D_{1} h \in(h, \theta) \text {. }
$$

We proceed by contradiction. Since $r \leq m-1$, contradicting 2.9 is equivalent to assume that $r \neq 0, m \geq r+2$ and that $D_{1} h$ is invertible. Since $m \geq r+2$, the right hand side of the equality 2.8 belongs to $\left(h^{r}, \theta\right)$. On the other hand, since $m>r>0$ and both $\gamma$ and $D_{1} h$ are invertible, the left hand side of 2.8 does not belong to $\left(h^{r}, \theta\right)$. This is a contradiction.

We now conclude our proof. The scheme $W_{\text {red }}$ is not $D_{1}$-invariant by hypothesis. First, we prove that this is equivalent to saying that $D_{1} h \notin(h, \theta)$. Recall that the ideal of $W_{\text {red }}$ is $(h, \theta)$, and that $D_{1}$ is tangent to $\Theta$ at all points of $W_{\text {red }}$ (by definition of $W$ ). On the other hand, $D_{1} h$ vanishes on $W_{\text {red }}$ if and only if $D_{1}$ is tangent to the locus $\{h=0\}$ at all points of $W_{\text {red }}$. Therefore, as $(h, \theta)$ defines $W_{\text {red }}$, the vector field $D_{1}$ is tangent to $W_{\text {red }}$ at all points of $W_{\text {red }}$ (i.e. $W_{\text {red }}$ is $D_{1}$-invariant) if and only if $D_{1} h \in(h, \theta)$.

Let us go back to 2.7. Since $\delta, \rho$ and $D_{1} h$ are invertible, $t \geq 1$. Since $r=t \geq 1$ and since $D_{1} h$ is invertible, 2.6 and 2.9 give $r=t=m-1 \geq 1$. It follows that $r+t=2(m-1) \geq m$ which proves $(\star)$.

$$
\text { q.e.d. }
$$

\section{Complements: an application to the non-degenerate case}

Let $(X,[\Theta])$ be an indecomposable principally polarized abelian variety. Assume that there exist points $a, b, c$ in $X$ such that $K(a), K(b)$ and $K(c)$ are distinct and collinear, where $K: X \rightarrow|2 \Theta|^{\star}$ denotes the Kummer morphism. By Fay's trisecant formula (see $[\mathrm{F}]$, p. 34) there exist non-zero constants $\alpha, \beta, \gamma$, such that

$$
\alpha \cdot \theta_{a} \cdot \theta_{-a}+\beta \cdot \theta_{b} \cdot \theta_{-b}+\gamma \cdot \theta_{c} \cdot \theta_{-c}=0,
$$

where $\theta_{x}:=\theta(z-x)$. First, one would like to prove the following 


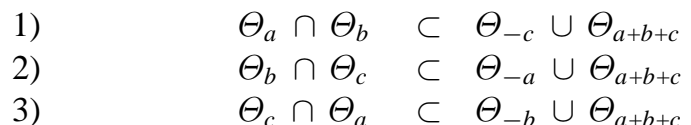

(the geometrical interpretation of each inclusion in 4 is that there exists a degenerate trisecant to the Kummer variety of $X$. More precisely, as $2 u=a-b$ and $v=u+b+c$, the inclusion 4.1 is equivalent to the inclusion $\Theta_{u} \cap \Theta_{-u} \subset$ $\Theta_{v} \cup \Theta_{-v}$ (via translation by $u+b$ ). This, in turn, is equivalent to the property that the line joining $K(u)$ and $K(v)$ is tangent to $K(X)$ at $K(u)$ and meeets $K(X)$ at $K(v)$ (see [D1]). We can easily prove that the inclusions in 4 hold provided that an extra hypothesis holds. Indeed, defining

$$
\begin{aligned}
& W_{a, b}:=\bigcup S \mid S \text { is a component of } \Theta_{a} \cap \Theta_{b}, S \not \subset \Theta_{-c} \cup \Theta_{a+b+c} \\
& W_{b, c}:=\bigcup S \mid S \text { is a component of } \Theta_{b} \cap \Theta_{c}, S \not \subset \Theta_{-a} \cup \Theta_{a+b+c} \\
& W_{c, a}:=\bigcup S \mid S \text { is a component of } \Theta_{c} \cap \Theta_{a}, S \not \subset \Theta_{-b} \cup \Theta_{a+b+c}
\end{aligned}
$$

one has the following:

Lemma 5. $\left(W_{a, b}\right)_{\mathrm{red}}=\left(W_{b, c}\right)_{\mathrm{red}}=\left(W_{c, a}\right)_{\mathrm{red}}$.

Proof. It suffices to prove that $\left(W_{a, b}\right)_{\text {red }} \subset\left(W_{b, c}\right)_{\text {red }}$. For this we need to prove that if $S$ is a component of $\Theta_{a} \cap \Theta_{b}$ such that $S \not \subset \Theta_{-c} \cup \Theta_{a+b+c}$, then there exists a component $Q$ of $\Theta_{b} \cap \Theta_{c}$ such that $Q \not \subset \Theta_{-a} \cup \Theta_{a+b+c}$, and moreover $S_{\text {red }}=Q_{\text {red }}$. Let $S$ be as above and let $p$ be a generic point of $S_{\text {red }}$. By Ein-Lazarsfeld's theorem $\Theta_{b}$ is non-singular along $S_{\text {red }}$, i.e. $h:=\theta_{b}$ is an irreducible element in the local ring $Q_{X, p}$. Since $S_{\text {red }}$ is reduced of codimension 2, there exists another irreducible element $k$ in $\mathscr{Q}_{X, p}$ such that the ideal defining $S_{\text {red }}$ at $p$ is $(h, k)$. Let $l, r, m, s, t$ be integers such that

$$
\begin{array}{lll}
\theta_{a+b+c} & \in & \left(k^{l}\right)-\left(K^{l+1}\right) \bmod (h) \\
\theta_{a} & \in & \left(k^{r}\right)-\left(K^{r+1}\right) \bmod (h) \\
\theta_{-a} & \in & \left(k^{m}\right)-\left(K^{m+1}\right) \bmod (h) \\
\theta_{-c} & \in & \left(k^{s}\right)-\left(K^{s+1}\right) \bmod (h) \\
\theta_{c} & \in & \left(k^{t}\right)-\left(K^{t+1}\right) \bmod (h) .
\end{array}
$$

By Fay's trisecant formula 3 we get $r+m=s+t$. Therefore $l+s<m$ if and only if $l+r<t$, i.e. $\theta_{-c} \cdot \theta_{a+b+c} \notin\left(h, k^{m}\right)$ if and only if $\theta_{-a} \cdot \theta_{a+b+c} \notin\left(h, k^{t}\right)$, i.e. $\left.\theta_{-c} \cdot \theta_{a+b+c}\right|_{s} \neq 0$ if and only if $\left.\theta_{-a} \cdot \theta_{a+b+c}\right|_{Q} \neq 0$.

q.e.d.

Lemma 5 implies that the inclusions in 4 are equivalent, and that the scheme $\left(W_{a, b}\right)_{\text {red }}$ is invariant under the translations $t_{a-b}, t_{b-c}, t_{c-a}$. To prove this, denote by $\rho_{x}$ the involution $X \rightarrow X, p \mapsto x-p$. Since both $\Theta_{a} \cap \Theta_{b}$ and $\Theta_{-c} \cup \Theta_{a+b+c}$ are $\rho_{a+b}$-invariant, the scheme $W_{a, b}$ is $\rho_{a+b}$-invariant, so $\left(W_{a, b}\right)_{\text {red }}$ is $\rho_{a+b}$-invariant. Similarly, $\left(W_{b, c}\right)_{\text {red }}$ is $\rho_{b+c}$-invariant and $\left(W_{c, a}\right)_{\text {red }}$ is $\rho_{c+a}$-invariant. By lemma 5 it follows that $\left(W_{a, b}\right)_{\text {red }}$ is $\left\langle\rho_{a+b}, \rho_{b+c}, \rho_{c+a}\right\rangle$ invariant. This implies our claim because $t_{a-b}=\rho_{a+c} \circ \rho_{a+b}$ and similarly for $t_{a-c}$ and $t_{b-c}$. 


\section{Concluding remark}

Let us go back to the list of hypotheses from the introduction, and assume that $K(X)$ has an honest trisecant. By the argument above, there exist "degenerate trisecants" (namely the inclusions in 4, and ii), hold), provided that $\Theta$ does not contain $\left\langle t_{a-b}, t_{a-c}\right\rangle$-invariant divisors. This extra hypothesis is exactly the one of Debarre in [D2]. There he gives a proof of a weaker version of Welters' conjecture. An argument similar to the one above can be used to find inflectionary trisecants (i.e. to show that $\mathrm{iii}$ ) holds) and eventually to apply Theorem 2 above, thereby reproving the result in [D2]. Unfortunately we are not able to improve the results in [D2]. Nevertheless, we think that the method of going from $i$ ) to ii), to $i i i)$, to the K.P. hypothesis has the advantage of avoiding the hierachies in [D2]. In some sense, it shifts some of the difficulties to the last step, namely the one involving the K.P., the proof of which has already been done (see [S], or $[\mathrm{AD} 2]$, or $[\mathrm{M}])$.

\section{References}

[A] E. Arbarello: Fay's trisecant formula and a characterization of Jacobian Varieties. Proceedings of Symposia in Pure Mathematics 46 (1987)

[AD1] E. Arbarello, C. De Concini: Geometrical aspects of the Kadomtsev-Petviashvili equation. L.N.M. 1451 (1990), 95-137

[AD2] E. Arbarello, C. DeConcini: Another proof of a conjecture of S. P. Novikov on periods of abelian integrals on Riemann surfaces. Duke Math. J. 54 (1987), 163-178

[D1] O. Debarre: Trisecant lines and Jacobians. Journal of Algebraic Geometry 1 (1992), 5-14

[D2] O. Debarre: Trisecant lines and Jacobians II. Preprint (alg-geom/9306007)

[EL] L. Ein, R. Lazarsfeld: Singularities of theta divisors, and the birational geometry of irregular varieties. Preprint (alg-geom/9603017)

[F] J. Fay: Theta Functions on Riemann Surfaces. L.N.M. 352 (1973)

[M] G. Marini: A geometrical proof of Shiota's theorem on a conjecture of S.P. Novikov. To appear

[S] T. Shiota: Characterization of Jacobian varieties in terms of soliton equations. Invent. Math. 83 (1986), 333-382

[W1] G. Welters: A criterion for Jacobi varieties. Ann. Math. 120 (1984), 497-504

[W2] G. Welters: On flexes of the Kummer vaiety. Proc. Kon. Ned. van Wetenschappen, series A, 86; Indagationes Math. 45 (1983), 501-520 